\title{
EL COMPROMISO ORGANIZACIONAL Y SU RELACIÓN CON ALGUNOS FACTORES DEMOGRÁFICOS Y PSICOLÓGICOS.
}

\author{
Fernando Arias Galicia ${ }^{1}$ \\ Daniel Varela Sosa ${ }^{2}$ \\ Alejandro Loli Pineda ${ }^{3}$ \\ María Quintana Otiniano 4
}

Se busca conocer la relación que existe entre el compromiso organizacional y algunas variables demográficas (estudiados por Mathieu y Zajac) en una muestra de 190 personas con una elevada educación, proveniente de diversas organizaciones y de variados tamaños. Se encontró correlación alta entre compromiso afectivo y satisfacción general con el trabajo, también entre compromiso afectivo y compromiso con el trabajo, igual que compromiso afectivo y claridad del rol. En cambio no fue significativa la relación con jerarquía del puesto.

Por otro lado, las correlaciones entre el compromiso de conveniencia y las demás variables resultaron inferiores, siendo la más alta con claridad del rol, seguida por satisfacción con el trabajo. En cambio ninguna de las variables demográficas denotó asociación importante con el compromiso de conveniencia. Ni el sexo ni el estado civil denotaron ejercer influencia alguna sobre el compromiso.

PALABRAS CLAVE: Compromiso, miembros, organizaciones, factores demográficos.

One looks for to know the relation that exists between the organizacional commitment and some demographic variables (studied by Mathieu and Zajac) in a sample of 190 people with a high education, originating of diverse varied organizations and so large. Was high correlation between affective commitment and general satisfaction with the work, also between affective commitment and commitment with the work, just as affective commitment and c1arity of the roll. However the relation with hierarchy of the position was not significant.

On the other hand, the correlations between the convenience commitment and the other variables were inferior, being highest with clarity of the ro1, followed by satisfaction with the work. However no of the demographic variables denoted important association with the convenience commitment. Neither sex nor the civil state denoted to exert influence some on the commitment.

KEY WORDS: Commitment, members, organizations, Demographic factors.

1. División de Estudios de Postgrado, Facultad de Contaduría y Administración, Universidad Nacional Autónoma de México. Profesor Honorario de la Universidad de San Martín de Porres, Lima, Perú. Apdo. Postal 70-589, Coyoacán, México, D.F., C.P. 04511, Correo electrónico: fagl@infose1.net.mx

2. Decano de la Facultad de Ciencias Administrativas y Relaciones Industriales, Universidad de San Martín de Porres, Lima, Perú.

3. Decano de la Facultad de Psicología, Universidad Mayor de San Marcos (Universidad, DECANA de América), Lima, Perú; correo electrónico: alolip@unmsm.edu.pe. Presidente de la Asociación de Facultades, Escuelas e Institutos de Psicología de América Latina (AFEIPAL).

4. Jefa del Instituto de Investigaciones de la Facultad de Ciencias Administrativas y Relaciones Industriales, Universidad de San Martín de Porres, Lima, Perú. 


\section{INTRODUCCIÓN}

Las organizaciones constituyen agrupamientos sociales creados y modificados constantemente para alcanzar objetivos específicos (Parsons, 1960 Blau y Scott, 1963). Al ser entendidas compuestas por múltiples personas, se plantea un problema fundamental ¿Cómo lograr que cada cual se involucre con los objetivos para los cuales fueron establecidos? Ya lo dice el refrán "Cada cabeza es un mundo". Entonces, uno de los problemas primordiales de las organizaciones consiste en alcanzar la unidad en cuanto a logro de objeticos tomando como base la pluralidad. En efecto ¿Cómo lograr la coordinación y, sobre todo, el esfuerzo continuados hacia las metas que, se supone, deben ser comunes a todos, sin importar los aspectos idiosincrásicos?

No es difícil apreciar la importancia del compromiso (como se definirá mas adelante) de los miembros de la organización para alcanzar la efectividad. Sin dicho empeño, será mas difícil para las organizaciones lograr la competitividad y el éxito. En la actualidad, y cada mas en el futuro, el compromiso de las personas con la organización constituirá parte trascendente del capital humano (ver Arias Galicia y Heredia Espinoza, 1999, para una descripción de dicho capital). Aún cuando todavía no se acostumbre especificar este rubro directamente de los estados financieros [la primera excepción está dada por la empresa escandinava de seguros y servicios financieros Skandia (Edvisson y Malone, 1998)], intuitivamente puede verse que el compromiso de los miembros de una organización puede redundar de manera positiva en la productividad y los resultados financieros.

Para confirmar lo anterior se citan cuatro pesquisas al respecto, de las muchas existentes. Arthur (1994) efectuó una de la investigación entre 30 pequeñas acererías; dividió los sistemas de Recursos Humanos en tendientes al control (en donde se colocaba énfasis en reducción de costos, adherencia de los empleados a las reglas especificas y las remuneraciones basadas en criterios mensurables) y tendientes al compromiso (en donde existían niveles altos de participación, capacitación en solución de problemas en grupo, acciones de socialización, capacitación, y remuneraciones más latas que el promedio); los resultados indicaron que en estas últimas empresas existían menores porcentajes de desperdicio y de rotación de personal así como mas eficiencia de los trabajadores.

Por su parte: Tsui, Pearce, Lyman y Trípoli (1997) encontraron también que las empresas cuyas políticas iban en el sentido de invertir en sus empleados y procura su bienestar obtenían mejores rendimientos.

Huselid (1995) aplicó una encuesta a 986 encuestadores de diversos ramos de actividad económica así como de diversos tamaños y encontró claras relaciones entre las acciones tendientes a incrementar la participación de los trabajadores, efectuar una cuidadosa selección de los nuevos empleados, capacitarlos compartir las utilidades, otorgar ascensos con base a los méritos, remuneraciones basadas en el desempeño ( o sea, aspectos motivacionales) y los resultados de las empresas: por cada desviación estándar en dichas acciones encontraron: disminución de la rotación en un $7.05 \%$, por cada empleado, $\$$ 27.044 de incremento en ventas, \$ 18.641 en el valor del mercado y \$ 3.814 en utilidades (Dólares americanos).

Igualmente Lau, Wing y Ho (2003), al efectuar un meta-análisis con 40 investigaciones publicadas incluyendo 42.359 sujetos, concluyeron que el ausentismo es prevalente en personas de sexo femenino jóvenes, con bajo nivel de salario y con baja satisfacción en el trabajo.

Así pues, se tienen indicios de la importancia de invertir en los trabajadores a fin de 
lograr su involucramiento. Por ello, es importante para las organizaciones conocer no solo el tipo sino también el grado de compromiso de sus miembros. Una de las consecuencias es intentar elevarlo, por las razones expresadas en párrafos previas; pero los medios para lograrlo, en conformidad con las explicaciones precedentes, son diferentes, de acuerdo al tipo de compromiso que desee alcanzar.

Por otro lado, el tema del compromiso ha sido investigado profusamente en los países desarrollados. La pesquisa científica al respecto en las naciones subdesarrolladas en muy magra. Sin embargo, con frecuencia se toman decisiones dentro de las organizaciones sin tomar en consideración los posibles efectos sobre dicho compromiso.

Por ejemplo, en muchos casos se han confundido la reingeniería y el adelgazamiento, con la reducción de las plazas de trabajo, dando lugar a despidos masivos, lo cual ha colocado mayor presión a los sobrevivientes pues las tareas se reparten entre, ellos. Los despidos masivos levan al subempleo o al engrosamiento de la economía informal, con algunas de las consecuencias indeseables: falta de seguridad en los ingresos, carencia de seguridad social, menores ingresos por impuestos en el sector Público, e, igualmente, menor demanda para productos y servicios, etc.

En muchas organizaciones se ha adoptado la modalidad del trabajo temporal, la cual va tomando mayor fuerza; una posible consecuencia es el compromiso disminuido de los trabajadores. En los empleos por temporada o por obra determinada: las organizaciones contratan a personas por líneas específicas. Ni las organizaciones ni los trabajadores esperan un empleo a lo largo del tiempo, como en épocas anteriores. Inclusive en Japón, país donde era tradicional el empleo de por vida, la situación está cambiando: cada vez es menos frecuente encontrar empresas oferentes de dicho.

Los trabajadores se convierten, así, en una pieza de una maquinaria, fácilmente substituibles, de acuerdo a las políticas de muchas organizaciones. Entonces, es de esperarse un compromiso menor hacia la organización. Las consecuencias de estas tendencias, tanto para las organizaciones como para los trabajadores, están por verse en Latinoamérica, en donde es magra la investigación al respecto, como se mencionó antes.

El compromiso es un tema que ha interesado desde hace varias décadas a los directivos y a los estudiosos del comportamiento humano en las organizaciones. Así, en 1977, Steers lo definió como “... la fuerza relativa de identificación y de involucramiento de un individuo con una organización». Propuso un modelo, el cual fue confirmado en una investigación entre científicos, ingenieros y empleados de un hospital, que plantea tres fuentes del compromiso:

- Características personales: auto-estima, necesidad de logro y de poder, edad, escolaridad, estrés, satisfacción con la vida, etc.; en resumen, se trata de la Personalidad y de los aspectos demográficos relativos.

- Características del trabajo: sentir el trabajo como un reto, identidad con la tarea, interacción con otros a discreción, retroinformación, etc. También aquí juega un papel importante la Personalidad.

- Experiencias en el trabajo: actitudes del grupo, percepción de la propia importancia en la organización así como las inversiones de tiempo, esfuerzo y otras efectuadas en la organización, expectativas de recompensas, confianza en la organización, etc. 


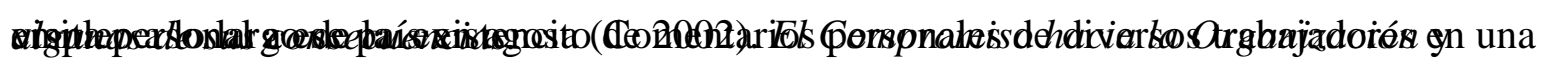

Las organizaciones pueden influir más fácilmente sobre las dos últimas fuentes. En cambio, transformar la personalidad sería mucho más difícil y cuestionable, desde un ángulo ético. La controversia se centraría respecto a la validez de la intromisión por parte de la organización en un aspecto central para el individuo. Al ingresar un nuevo elemento a la organización, lleva consigo sus atributos y facultades humanas así como un aprendizaje social.

Volviendo a los conceptos, desde el ángulo del comportamiento organizacional, el compromiso puede considerarse también como la lealtad o apego de un individuo hacia la organización que lo emplea (Bozeman y Perrewé, 2001).

Sin embargo, la existencia de un contrato escrito o verbal de trabajo no implica automáticamente el compromiso de ambas partes. Como es fácil apreciar, la formalización de un contrato obliga a las partes. Sin embargo, no necesariamente pueden especificarse todas las facetas del trabajo y el salario en un contrato de trabajo.

Desde hace tiempo se ha establecido una distinción entre el contrato de trabajo legal y el psicológico (Rousseau, 1990, 1995). Mientras una persona puede estar obligada formalmente a desempeñar una labor dentro de una organización, no necesariamente se compromete o liga afectivamente a la misma. Puede trabajar con disgusto y sentir desprecio hacia aquélla, esperándose un rendimiento menor así como otras posibles consecuencias: estrés, conflictos obrero-patronales, ausentismo, abandono de la organización, robos de materiales, dinero o bienes, etc. En este caso, los costos de todo tipo (emotivos, económicos, de salud, etc.) tanto de la organización como de la persona se verán incrementados.

Un individuo respecto a las obligaciones recíprocas. [Estas] creencias se convierten en contractuales cuando el individuo cree que ... está obligado con el empleador a proporcionarle ciertas contribuciones [por ejemplo: trabajo intenso, lealtad, sacrificios] a cambio de ciertos incentivos [verbigracia: paga elevada, seguridad en el trabajo]».

Por tanto, si las personas son generadoras, en gran parte, de los resultados organizacionales, entonces los empleados comprometidos constituyen una ventaja competitiva, igualo más que el conocimiento y las habilidades y el capital fijo (maquinaria, edificios, etc.) o el financiero (Recuérdense las cuatro investigaciones citadas previamente). Así, resulta trascendente para las organizaciones conocer cuáles son algunos de los factores asociados con el mencionado compromiso; sobre algunos podrá influir y otros ya son propiedad de las personas, como se mencionará más adelante.

Así pues, solamente si existe un contrato psicológico por parte de los trabajadores, vale decir, un compromiso, pondrán al servicio de la organización su cabal esfuerzo, así como sus facultades humanas (iniciativa, creatividad, etc.). Por tanto, el compromiso constituye una parte trascendente en la efectividad y la competitividad de las organizaciones.

Es de notarse que en las conceptuaciones anteriores, el compromiso es visto como una entidad única, sin elementos constitutivos.

\section{Los tres componentes del compromiso}

Por su parte, Meyer y Allen (1991) definieron el compromiso como un estado psicológico que caracteriza la relación entre una persona y una organización, la cual presenta consecuencias respecto a la decisión para continuar en la organización o dejarla. Estos autores, en contra de la corriente inicial de considerar al compromiso como un constructo único, propusieron una división del compromiso en tres componentes: afectivo, de continuidad y normativo. 
El Compromiso Afectivo toma como base el deseo y se refiere a los lazos emocionales que las personas forjan con la organización al percibir la satisfacción de sus necesidades (especialmente las psicológicas) y expectativas; por ende, disfrutan de su permanencia en la organización.

En el segundo componente, el Compromiso de Conveniencia o de Continuidad (fundamentado en la necesidad), es muy posible encontrar una conciencia de la persona respecto a las inversiones en tiempo y esfuerzo que se perderían en caso de dejar la organización, o sea, de los costos (financieros, físicos. psicológicos) en los cuales se incurriría al retirarse, o de las pocas posibilidades para encontrar otro empleo. Se refleja aquí una faceta calculadora, pues se refiere a la prosecución de inversiones (planes de pensiones, primas de antigüedad, aprendizaje, etc.) acumuladas y resultantes de la pertenencia a la organización. Dejarla resulta cada vez más costoso y, por lo tanto, se crea un «compromiso por omisión» (Becker, 1960). Puede suponerse que un elevado índice de desempleo en una sociedad acarreará un mayor compromiso de conveniencia (Vandenberghe,1996).

En el tercer componente, o sea el compromiso normativo (deber), se encuentra la creencia en la lealtad a la organización (sentido moral), quizá por recibir ciertas prestaciones (por ejemplo, capacitación o pago de colegiaturas), conducente a un sentido del deber de proporcionar una correspondencia. Este aspecto va en concordancia con la teoría de la reciprocidad: quien recibe algún beneficio adquiere el precepto moral interno de retribuir al donante (Gouldner, 1960).

Es posible pensar que el compromiso individual acarrea ciertas consecuencias tanto favorables como desfavorables para las organizaciones. En el primer punto se esperaría una intención de permanencia, es decir, continuar manteniendo los nexos con la organización. Como es sabido, la rotación de personal implica costos de diversos tipos para las organizaciones (reclutamiento, selección, capacitación, errores, accidentes. etc.). Así pues, se incluyó en esta investigación el propósito mencionado. Aquí es pertinente recordar que las intenciones constituyen la antesala de los comportamientos. de acuerdo con la Teoría de Azjen y Fishbein $(1977,1980)$.

En cuanto a la falta de compromiso por parte de los miembros de la organización, además de la retirada del empleo, pueden citarse: el ausentismo y los robos. El primero acarrea costos para la organización pues pueden verse interrumpidos los flujos de trabajo, además de la contratación de substitutos, la disminución en la calidad, etc. Los hurtos pueden ser minúsculos y pasar casi desapercibidos y, sin embargo, si muchos trabajadores caen en ellos, las pérdidas pueden ser cuantiosas. Por ejemplo, se ha calculado que, en los Estados Unidos. las cifras fluctúan entre 6 y 200 millones de dólares al año (Green, citado por Winbush y Dalton, 1997); dichas substracciones pueden darse ante la sensación de que la organización no está comprometida con sus empleados.

Además de lo anterior, en el lado negativo, un compromiso muy elevado puede significar la inflexibilidad de los miembros de la organización respecto a las transformaciones en la misma, así como rechazo a las innovaciones y, por ende, falta de adaptabilidad (Randall, 1987). En caso de presentarse esta situación, la competitividad podría resultar afectada de forma contraproducente.

Por otro lado, el compromiso acendrado puede dar lugar al fenómeno denominado «persistencia en una acción fallida», el cual consiste en perseguir un curso de actuación, invirtiendo cada vez más recursos aún ante el fracaso, con la esperanza de que al final los resultados sean beneficiosos (Brockner, 1992; Schaubreck y Williams, 1993; Staw y Roos, 1988).

Como antes se mencionó, aunque no se acostumbra adjuntar datos sobre la motivación de los miembros de la organización (y otros aspectos relevantes sobre el factor humano) en 
los estados financieros, ya existen organizaciones precursoras en este asunto (Ver a Edvinsson y Malone, 1998). Por ende, en un futuro, dada la intensa competencia en la economía global, el compromiso de las personas hacia su organización será un aspecto de importancia en la toma de decisiones dentro de las empresas.

\section{Aspectos demográficos}

Ahora bien, existen factores que no pueden ser modificados por la organización, como antes se apuntó, sino son elementos portados por las personas al ingresar. Entre ellos, aparte de la personalidad se encuentran los aspectos demográficos.

Mathieu y Zajac publicaron una revisión de la literatura publicada al respecto desde 1967 hasta 1987. No tomaron la clasificación propuesta por Meyer y Allen (1991) sino dividieron el compromiso en dos facetas: actitudinal y calculador. El primero lo refirieron a «... la intensidad relativa de identificación e involucramiento de un individuo con una organización particular». Conceptuaron al Compromiso Calculador como la liga entre el individuo y la organización debida a las inversiones del primero en la segunda en forma de tiempo, planes de pensión, etc.; por tanto, la persona percibe ciertos «costos ocultos» y no puede darse el lujo de abandonarla. Nótese el paralelismo con el compromiso afectivo y el de continuidad propuestos por Meyer y Allen (1991).

Entre las principales conclusiones de

Mathieu y Zajac (1990) destacan las siguientes:

- La edad mostró más altas correlaciones con el compromiso actitudinal que con el calculador.

- Las mujeres denotaron más alto compromiso actitudinal, aunque las diferencias con el calculador resultaron pequeñas.

- La educación exhibió una tendencia negativa con ambos tipos de compromiso, aunque fue más intensa con el actitudinal.

Las personas casadas mostraron más alto compromiso. Los autores no encontraron investigaciones suficientemente numerosas como para distinguir entre el actitudinal y el calculador.

- La antigüedad en el puesto denotó mayor correlación positiva con el compromiso actitudinal; mientras la antigüedad en la organización lo hizo con el compromiso calculador.

- La jerarquía del puesto mostró relación positiva con el compromiso (aunque no especifican de qué tipo).

- Otro de los hallazgos va en el sentido de una relación negativa entre la ambigüedad del rol y el compromiso.

- Las correlaciones entre las características personales y el compromiso resultan relativamente pequeñas.

- El compromiso con el trabajo también dio una correlación positiva con el compromiso organizacional.

- El compromiso actitudinal mostró asociaciones más altas con la satisfacción general en el trabajo y la satisfacción con el trabajo en sí.

La investigación delineada en la presenta investigación a grandes rasgos pretende verificar si en el Perú se encuentran resultados semejantes a los descritos por los dos autores últimamente mencionados. 


\section{HIPÓTESIS}

La hipótesis principal estribó en encontrar resultados semejantes a los hallazgos de Mathieu y Zajac (1990) mencionados en los párrafos precedentes, con las variables tomadas en consideración en el presente trabajo, las cuales se definen más abajo. En otras palabras, se formuló la hipótesis nula de falta de asociación entre las variables cuantitativas. Por otro lado, la hipótesis nula en cuanto a las variables nominales (sexo y estado civil) consistió en esperar semejanzas en las medias del Compromiso Afectivo y del de Conveniencia entre dichos grupos.

\section{MÉTODO}

\section{Participantes:}

La muestra estuvo constituida por 190 personas, de las cuales 54\% fueron del sexo masculino y $46 \%$ del femenino. Las edades se distribuyeron de la siguiente manera: 21 a 30 años, 38\%; 31 a 40, 34\%; 41 a 50, $21 \%$; y más de 50, 7\%. En lo tocante al estado civil, los porcentajes resultaron: solteros, $50 \%$; casados, $45 \%$; divorciados, $2 \%$; y otros, $3 \%$ (sin embargo, no todos los participantes incluyeron este dato, por lo cual la muestra se redujo a 167 casos en cuanto al análisis de esta variable; en virtud de los bajos porcentajes de algunas categorías, los divorciados y los viudos se sumaron a los solteros, mientras los convivientes se adjuntaron a los casados). Respecto a la escolaridad: hasta Bachillerato, 30\%; Licenciatura, 36\%; y Postgrado, 34\%. El ramo de actividad económica en donde laboraban los participantes fue: servicios, 12\%; industria, 15\%; educación, 33\%; salud, $10 \%$; consultaría, $4 \%$; gobierno y otros, $23 \%$. El tamaño de las organizaciones resultó estar representado de la siguiente manera: menos de 25 empleados, 22\%; de 26 a 50, 12\%; 51 a $100,16 \% ; 101$ a 200, $11 \% ; 201$ a 500,15\%; Y más de 500, $24 \%$.

Puede verse entonces, que se trata de una muestra de conveniencia y de personas con elevada educación mayoritariamente, proveniente de diverso tipo de organizaciones y de varios tamaños. Las mujeres representan casi la mitad de la muestra.

\section{Instrumentos y variables:}

Se preparó un cuestionario incluyendo reactivos correspondientes a:

- Compromiso (Meyer y Allen, 1991), aunque sólo se consideró el Afectivo y el de Conveniencia (definidos anteriormente).

- Satisfacción General con el Trabajo (4 reactivos empleados por Eisenberger, et. al. 1997). Se refiere a la respuesta afectiva dada por la persona a su puesto (Smith, 1976).

- Claridad del Rol (Brown y Leigh, 1996). Nótese que en los resultados de Mathieu y Zajac (1990) se habla de ambigüedad del rol, mientras en este trabajo se emplea su contrapartida: Claridad del Rol. Según los autores primeramente citados, esta variable se refiere a la diafanidad de las expectativas así como a las normas de trabajo consistentes y que, además, pueden predecirse para crear un ambiente seguro de trabajo.

- Compromiso con el Trabajo (6 reactivos tomados del cuestionario de Lodahl y Kejner, 1965). Se refiere al grado en el cual la persona ha acogido los valores respecto a la bondad de sus labores y la importancia de las mismas para su propia valía. 
Se adicionó un formato para recabar los datos demográficos enunciados a continuación:

- Sexo (condición biológica que determina el papel jugado en la reproducción).

- Edad (lapso en el cual ha vivido una persona, medido en años).

- Escolaridad (Gradación alcanzada en los estudios formales).

- Estado civil (situación legal relativa al matrimonio).

- Antigüedad en la organización (lapso de permanencia en la misma, medida en años).

- Antigüedad en el puesto (período en el cual una persona ha desempeñado el mismo trabajo, medido en años).

- Jerarquía organizacional (responsabilidad en cuanto a la supervisión de otras personas así como de recursos en una organización). Procedimiento:

Los cuestionarios se aplicaron primordialmente durante clases de postgrado en instituciones educativas. También se administraron a personas dentro de algunas organizaciones.

\section{Procesamiento de datos:}

Se empleó el SPSS (Statistical Package for the Social Sciences), versión 11.5. Se capturaron dos veces los datos, por personas independientes, y se sometieron a un proceso de limpieza y verificación.

A fin de verificar los resultados obtenidos por Mathieu y Zajac (1990) se calcularon las correlaciones entre las variables medidas al menos en una escala ordinal (Pearson y Kendall; los resultados entre ambos tipos fueron semejantes y por eso se incluyen sólo las primeras pues son más conocidas y con mayor facilidad en su interpretación).

En cuanto a las dos variables demográficas nominales (sexo y estado civil) se decidió aplicar un análisis de la varianza, introduciendo las dos al mismo tiempo.

Se decidió utilizar el criterio de $\mathrm{p}=<.05$ para rechazar las hipótesis nulas.

\section{RESULTADOS}

En la tabla 1 aparecen, en primer lugar, las medias y las desviaciones estándar del Compromiso Afectivo y el de Conveniencia, y en seguida, las correlaciones con las variables demográficas de interés (Edad, Escolaridad, Antigüedad en la Organización, Antigüedad en el Puesto, y Jerarquía del Puesto) así como con Claridad del Rol, Compromiso con el Trabajo y Satisfacción General con el Trabajo. Puede verse que la media más elevada correspondió al Compromiso Afectivo, con una menor variabilidad. La correlación más alta se presentó entre el Compromiso Afectivo y la Satisfacción General con el Trabajo, seguida por la existente entre aquélla y el Compromiso con el Trabajo. En tercer lugar estuvo la asociación entre la primera variable mencionada y la Claridad del Rol. Por ende, se rechazó la hipótesis nula de carencia de asociación entre las variables mencionadas.

En cambio, no hubo significación estadística con la Jerarquía del Puesto, en contra de lo esperado. Las demás correlaciones se presentaron en el sentido esperado por la teoría, aunque no alcanzaron la significación estadística, dando lugar a la aceptación de las hipótesis nulas respectivas.

Es importante subrayar que las correlaciones entre el Compromiso de Conveniencia y las demás variables resultaron inferiores a las presentadas con el Compromiso tratado en el párrafo precedente. Aquí la correlación más alta fue con la Claridad del Rol, seguida por la 
Satisfacción con el Trabajo. Ninguna de las variables demográficas denotó asociación importante con el Compromiso de Conveniencia, dando lugar a la aceptación de las hipótesis nulas correspondientes.

En la Tabla 2 se incluyen las medias aritméticas de los dos tipos de Compromiso en relación al Sexo y el Estado Civil. En cuanto a la variable primera, la $\mathrm{F}$ para el Compromiso Afectivo resultó de 2.369 con una probabilidad de 0.126 , mientras para el de Continuidad fue de 0.534 con una probabilidad de 0.466 . Por lo que toca a la variable estado civil, los valores respectivos fueron $0.202(\mathrm{p}=0.654)$ y $0.485(\mathrm{p}=0.487)$. Las interacciones tampoco resultaron significativas. Por tanto se acepta la hipótesis nula de igualdad entre las medias de ambos tipos de compromiso; en otras palabras ni el sexo ni el estado civil denotaron ejercer influencia alguna sobre el compromiso.

\section{DISCUSIÓN}

La mayor parte de los resultados concuerdan con los hallazgos de Mathieu y Zajac (1990). En particular, la edad mostró correlación más alta con el Compromiso Afectivo que con el de Conveniencia; la Antigüedad en el Puesto denotó mayor asociación con el Afectivo, al igual que la Jerarquía (aunque en este caso no resultó significativa). También se corroboró otro aspecto: las correlaciones con los datos demográficos son relativamente pequeñas. En contra de lo esperado, la Escolaridad mostró una asociación positiva con el Compromiso, especialmente con el Afectivo. Quizá esta diferencia con los hallazgos de Mathieu y Zajac (1990) se deba a los aspectos culturales: mientras la investigación de dichos autores se efectuó en los países desarrollados (en donde imperan los valores individualistas y racionalistas) la cultura peruana es de mayor convivencia y emotiva (Hofstede, 1980); quizá las personas en el primer tipo de cultura están más interesadas en el propio desarrollo y los consecuentes resultados, lo cual se incrementa con la escolaridad. Este es un punto digno de investigación más detenida.

Merecedoras de especial atención son las correlaciones entre la Claridad del Rol, el Compromiso con el Trabajo y la Satisfacción General con el Trabajo: no sólo resultaron positivas sino también fueron las más elevadas, confirmando así los hallazgos de Mathieu y Zajac (1990). Aquí es importante señalar que estos factores tomaron preeminencia sobre los demográficos; es decir, las actitudes provenientes de las experiencias en la organización, especialmente la Satisfacción con el Trabajo, presentaron una importancia mayor: las acciones de la organización parecen mostrar mayor influencia sobre el Compromiso que los factores demográficos.

La idea anterior se ve reforzada por los resultados concernientes al sexo y al estado civil, los cuales en ningún caso mostraron significación. No obstante, se confirmó que las mujeres mostraron mayor puntuación en cuanto al Compromiso Afectivo. También se verificó el mayor Compromiso, tanto Afectivo como de Conveniencia, de las personas casadas, si bien las diferencias son mínimas.

Estos hallazgos indican la importancia de las acciones de la organización en cuanto a los aspectos subjetivos de Satisfacción y Compromiso. Igualmente, implican la trascendencia de los aspectos organizacionales para lograr el compromiso, el cual puede acarrear consecuencias positivas para la organización, como se mencionó en la primera parte de este trabajo.

Es necesario apuntar las limitaciones de la presente investigación. Como sucede con frecuencia en las pesquisas en las organizaciones, la muestra fue de conveniencia, y limitada a la ciudad de Lima, además de contar con un gran porcentaje de personas con grado elevado de escolaridad; por ende no es necesariamente representativa de los trabajadores de todo el país. Serán necesarias investigaciones adicionales para corroborar si en otras ciudades de la nación y con una dispersión más amplia de educación formal se 
presentan resultados semejantes.

Igualmente, se precisa indagar la contribución de la personalidad de cada individuo (la cual no se analizó en la presente investigación) al Compromiso y la Satisfacción. Por ejemplo, en una metaanálisis de 163 muestras independientes (tomadas en su mayoría de países industrializados, especialmente de los Estados Unidos), Judge, Heller y Mount (2002) encontraron correlaciones entre los cinco grandes factores de la Personalidad y la Satisfacción con el Trabajo: -0.29 con Neuroticismo, 0.25 con Extraversión, 0.02 con Apertura a Nuevas Experiencias, 0.17 con Deseo de Agradar y 0.26 con Escrupulosidad, denotando así una disposición individual al respecto. En otra investigación (Judge, Bono y Locke, 2000), se encontró también que los rasgos de personalidad (auto-estima, autoeficacia, locus de control y bajo neuroticismo) estaban asociados a la satisfacción en el trabajo, inclusive después de 30 años; en efecto, estos autores tomaron datos de niños evaluados, como parte de un estudio longitudinal, por el Instituto de Desarrollo Humano de la Universidad de California, Berkeley, y después (cuando habían llegado al rango de 41 a 50 años) les pidió responder a un cuestionario sobre Satisfacción en el Trabajo. Los resultados dieron la misma tónica: los rasgos de personalidad estaban asociados a la satisfacción, aunque se presentó la mediación por parte de la complejidad del trabajo. En otras palabras, parece existir una predisposición individual para comprometerse, lo cual, aunado a las experiencias tenidas en la organización iría en favor de un mayor compromiso.

Indudablemente, existe otra serie de variables incidentes sobre la satisfacción y el compromiso (entorno económico y desempleo, cultura y clima organizacional, habilidades de las personas, orientación vocacional, etc.). Investigaciones posteriores deberán indicar la importancia relativa de estos factores. 


\section{REFERENCIAS BIBLIOGRÁFICAS}

Arias Galicia, F. (1972). Administración de Recursos Humanos. México:

Trillas. 1 a. Ed.

Arias Galicia, F. y Heredia Espinosa, V. (1999). Administración de Recursos Humanos para el Alto Desempeño. México: Trillas.

Ajzen, I. y Fishbein, M. (1977). AttitudeBehavior Relations: A theoretical analysis and Review of Empirical Research. Psychological Bulletin, 4,888-918.

Ajzen, I. y Fishbein, M. (1980).

Understanding Attitudes and Predicting Social Behavior:

Actitudes, lntentions, and Perceived Behavioral Control.

Englewood Cliffs. NJ.: PrenticeHall.

Arthur, LB. (1994). Effects of Human Resource Systems on Manufacturing Performance and Turnover. Academy of Management Journal, 37, 670-687.

Becker, H.S. (1960). Notes on the concept of commitment. American journal of sociology, 66, 32-42.

Blau, P.M. y Scott, WR. (1963). Formal organizations: a comparative approach. Londres: Routledge.

Bozeman, D.P \& Perrewé, P.L. (2001). The Effect of ltem Content Overlap on Organizational Commitment Questionna i re - Turnover Cognitions Relationships. Journal of Applied Psychology, 86, 161-173.

Brockner, J. (1992). The escalation of commitment to a failing course of action: toward theoretical progress. Academy of Management Review, 17, 39-62.

Brown, S.P. y Leigh, T.W (1996). A new look at psychological climate and its relationships to job involvement, effort, and performance. Journal of applied psychology, 81,358-367.

Edvinsson, L. y Malone, M.S. (1998). El capital intelectual. Bogotá: Norma.

Eisenberger, R., Cummings, J, Armeli, S. y Lynch, P (1997). Perceived Organizational Support, Discretionay Treatment, and Job Satisfaction. Journal of Applied Psychology, 82, 812-820.

Gouldner, A.W (1960). The norm of reciprocity: a preliminary statement. American Sociological Review, 25,161-178.

Hofstede, G. (1980). Culture's consequences: international differences in work related values. Beverly Hills, Ca.: Sage.

Huselid, M. A. (1995). The Impacr of Human Resources Management Practices on Turnover, Producrivity ands Corporate Financial Performance. Academy of Management Journal, 38, 635-672.

Judge, T.A., Bono, J.E. y Locke, EA (2000). Personality and Job Satisfaction:

The Mediating Role of 10b Characteristics. Journal of Applied Psychology, 85, 237-249.

Judge, T A, Heller, D. y Mount, M. K. (2002). Five-Factor Model of Personality and Job

Satisfaction; A MetaAnalysis. Journal of Applied Psychology, 87, 530-541.

Lau, V.L.S , Wing, TA y Ho, J.M.C. (2003) A qualitative and quantitative review of antecedents of counterproductive behavior in organizations. Journal of Business and Psychology, 18, 73-100.

Lodahl, T. y Kejner, M. (1965). The definition and measurement of job involvement. Journal of Applied Psychology, 49, 24-33.

Mathieu, J.E. y Zajac, D.M. (1990). A Review and Meta-Analysis of the Antecedents, Correlates and Consequences of Organizational Commitment. Psychological 
Bulletin, 208, 272-294.

Meyer, 1P. y Allen, Natalie J. (1991). A three-component conceptualization of organizational commitment. Human Resource Management Review, 1, 61-98.

Parsons, T (1960). Structure and process in modem societies. Glencoe, III.: Free Press.

Randall, D.M. (1987). Commitment and the organization: the organization man revisited. Academy of Management Review, 12, 460-471.

Rousseau, D.M. (1990). New hire perceptios or their own and their employer's obligations: a study of psychological contracts. Journal of Organizational Behavior, 11, 389-400.

Rousseau, D.M. (1995). Psychological contracts in organizations: understanding written and unwritten agreements. Thousan Oaks: Sage.

Schaubreck, J. y Williams, S. (1993). Type A Behavior Patlern and Escalating Commitment. Journal of Applied Psychology, 78, 862867.

Smith, P.C .. (1976). Desarrollo de un método para medir la satisfacción en el trabajo: los estudios de Comell. En Fleishman, E. A. Y Bass, A R. (1976). Estudios de psicología industrial y del personal. México: Trillas.

Staw, B.M. y Roos, J. (1988). Good money after bad: why do we be come overly committed to losing projects. Psychology Today, 22, 30-33.

Steers, R.M. (1977). Antecedents and outcomes of organizational commitment. Admnistrative Science Quarterly, 22, 46-56.

Tsui, A.S. , Pearce, L.J., Lyman, W.P. y Trípoli, A.M. (1997). Altemative Approaches to the EmployeeOrganization Relationship: Does Investment in Employees Pay Off? Academy of Management Journal, 40, 1089-1121.

Vandenberghe, C. (1996). Assessing organizational commitment in a Belgian context: evidence for the three-dimensional model. Applied psychology: an international review, 45, 371-386.

Wimbush, J.C. y Dalton, D.R. (1997). Base Rate for Employee Theft: Convergence of Multiple Methods. Journal of Applied Psychology, 82, 756-763. 
Tabla I. Correlaciones (Pearson) entre dos tipos de Compromiso y las variables indicadas.

\begin{tabular}{|c|c|c|}
\hline Variable & $\begin{array}{l}\text { Compromiso afectivo } \\
x=4.87 \\
S=1.13\end{array}$ & $\begin{array}{l}\text { Conveniencia } \\
x=4.46 \\
s=1.26\end{array}$ \\
\hline Edad & $.204^{\star \star}$ & .058 \\
\hline Escolaridad & $.171^{*}$ & .070 \\
\hline Antigüedad en la Org. & .090 & .065 \\
\hline Antigüedad en puesto de jerarquía & $.164^{*}$ & .062 \\
\hline Claridad del rol & .138 & .094 \\
\hline Compromiso con trabajo & $.411^{\star *}$ & $.209^{\star *}$ \\
\hline \multirow[t]{2}{*}{ Satisfacción general con el trabajo } & $.570^{* *}$ & $.145^{\star}$ \\
\hline & $.621^{* *}$ & $.199^{* *}$ \\
\hline
\end{tabular}

Tabla 2. Medias aritméticas de dos tipos de compromiso

\begin{tabular}{|c|c|c|c|c|c|}
\hline \multicolumn{6}{|c|}{$n=167$} \\
\hline \multirow{10}{*}{ AFECTIVO } & Sexo & $\begin{array}{l}\text { Estado civil } \\
\text { recodificado }\end{array}$ & Media & Desv. Tịp. & $\mathrm{N}$ \\
\hline & \multirow[t]{3}{*}{ Femenino } & Solteros & 4.8678 & 1.23752 & 59 \\
\hline & & Casados & 4.9946 & 1.20530 & 31 \\
\hline & & Total & 4.9115 & 1.22122 & 90 \\
\hline & \multirow[t]{3}{*}{ Masculino } & Solteros & 4.6414 & .98337 & 33 \\
\hline & & Casados & 4.6742 & .93841 & 44 \\
\hline & & Total & 4.6602 & .95167 & 77 \\
\hline & \multirow[t]{3}{*}{ Total } & Solteros & 4.7866 & 115241 & 92 \\
\hline & & Casados & 4.8067 & 1.06108 & 75 \\
\hline & & Total & 4.7956 & 110907 & 167 \\
\hline \multirow[t]{9}{*}{ CONVENIENCIA } & \multirow[t]{3}{*}{ Femenino } & Solteros & 4.2627 & 1.41279 & 59 \\
\hline & & Casados & 4.5645 & 1.11677 & 31 \\
\hline & & Total & 4.3667 & 1.31983 & 90 \\
\hline & \multirow[t]{3}{*}{ Masculino } & Solteros & 4.2879 & .99495 & 33 \\
\hline & & Casados & 4.2561 & 112757 & 44 \\
\hline & & Total & 4.2697 & 1.06602 & 77 \\
\hline & \multirow[t]{3}{*}{ Total } & Solteros & 4.2717 & 1.27295 & 92 \\
\hline & & Casados & 4.3836 & 112596 & 75 \\
\hline & & Total & 4.3220 & 1.20688 & 167 \\
\hline
\end{tabular}

